\title{
Comparison between measurements, simulations, and theoretical predictions of the extraction kicker transverse dipole instability in the Spallation Neutron Source
}

\author{
J. A. Holmes, ${ }^{1}$ S. Cousineau, ${ }^{1}$ V. Danilov, ${ }^{1}$ and L. Jain ${ }^{2}$ \\ ${ }^{1}$ Oak Ridge National Laboratory, Oak Ridge, Tennessee 37831-6461, USA \\ ${ }^{2}$ University of Wisconsin, Madison, Wisconsin 53715-1007, USA
}

(Received 4 June 2010; published 27 July 2011)

\begin{abstract}
Occasionally, it is possible to bring together experiment, theory, and simulation in detail. Such an occasion occurred during a high intensity beam physics study in the Spallation Neutron Source (SNS). A transverse dipole instability in the vertical direction has been observed in the accumulator ring for a coasting beam that was stored for 10000 turns. This instability was observed at a beam intensity of about $12 \mu \mathrm{C}$ and was characterized by a frequency spectrum peaking at about $6 \mathrm{MHz}$. The probable cause of the instability is the impedance of the ring extraction kickers. We carry out here a detailed benchmark of the observed instability, uniting an analysis of the experimental data, a precise ORBIT code tracking simulation, and a theoretical estimate of the observed beam instability.
\end{abstract}

DOI: 10.1103/PhysRevSTAB.14.074401

PACS numbers: 41.85.Ja, 41.75.Ak, 52.59.Wd

\section{INTRODUCTION}

The Spallation Neutron Source (SNS) accelerator consists of a $1 \mathrm{GeV} \mathrm{H}^{-}$linear accelerator followed by an accumulator ring of length 248 meters. At design intensity, a $1 \mathrm{~ms}$ pulse is stripper-foil injected into the accumulator ring, accumulated for approximately 1000 turns, and then extracted in a single turn for delivery to a liquid mercury target. During accumulation, the stored beam reaches an intensity of $1.5 \times 10^{14}$ protons, making it the most intense proton beam to date. The SNS accumulator ring consists of four $90^{\circ}$ arcs separated by four straight sections. Each arc is an achromatic focusing-defocusing (FODO) lattice, while each straight section contains a pair of doublets for focusing. Each straight section was designed to accommodate an insertion for a specific function. These functions are injection, collimation, extraction, and rf bunching, respectively. The ring is typically operated with bare tunes of $\nu_{x}=6.23$ and $\nu_{y}=6.20$.

Because the SNS ring is required to operate at this extremely high beam intensity, transverse instabilities have been a concern. Early estimates predicted that the extraction kickers would present the dominant ring impedance. Consequently, they were carefully designed to minimize that impedance. Even so, during the design of SNS, a broad study of transverse stability was conducted. The approach was twofold: we studied analytic coasting beam models $[1,2]$ in the SNS parameter regime and applied the results of these studies to benchmark [3] the transverse stability model in the ORBIT code [4]. With this confirmation of the accuracy of ORBIT, we then carried out stability

Published by the American Physical Society under the terms of the Creative Commons Attribution 3.0 License. Further distribution of this work must maintain attribution to the author(s) and the published article's title, journal citation, and DOI. calculations for realistic bunched beams obtained during injection. For comparison with the coasting beam results, these latter calculations were first done with single harmonic impedances. Finally, transverse stability was calculated for the full injection process using the measured values of the dominant extraction kicker impedance [5]. These studies predicted that, under normal operation with bunched beams, SNS would be stable. However, for coasting beams the instability threshold is much lower. This is all that could be concluded prior to the actual operation of SNS.

During the power ramp-up of SNS, some accelerator physics shifts were set aside for the study of high intensity ring stability issues. Under normal operating conditions with bunched beams, stable beams have been achieved in excess of $1.55 \times 10^{14}$ protons, or $25 \mu \mathrm{C}$. The bunchedbeam simulations predict stability at this intensity. In order to induce instabilities, a number of measures have been taken to reduce Landau damping. The ring rf buncher voltages and beam chopper settings were modified, or sometimes turned off altogether, so that coasting beams could be accumulated. Also, the chromatic sextupoles were activated in order to zero the ring chromaticity. In such cases, three independent instabilities have been observed. The frequency signatures of these instabilities strongly suggest (1) a broad electron cloud instability in the $20 \rightarrow$ $100 \mathrm{MHz}$ range, (2) a low frequency resistive wall instability at $\sim 100 \mathrm{kHz}$, and (3) a transverse (extraction kicker) impedance-induced instability in the $4 \rightarrow 10 \mathrm{MHz}$ range. The observations of these instabilities have been discussed in Refs. [6,7], and preliminary simulation results were shown in Refs. [8-10].

This paper treats the extraction kicker instability by bringing together the experimental results and theoretical estimate reported in Ref. [6] and a detailed simulation of the experiment using the ORBIT code [4]. Section II describes the extraction kickers in the SNS ring, together with 
TABLE I. SNS ring parameters.

\begin{tabular}{|c|c|c|c|c|}
\hline Parameter & Units & Design & Present production & Instability \\
\hline Circumference & meters & 248 & 248 & 248 \\
\hline Kinetic energy & $\mathrm{GeV}$ & 1.0 & 0.925 & 0.860 \\
\hline Bunch population & $\times 10^{14}$ protons & 1.5 & 1.0 & 0.77 \\
\hline$\gamma$, Relativistic gamma & & 2.066 & 1.986 & 1.917 \\
\hline$\beta$, relativistic beta & & 0.875 & 0.864 & 0.853 \\
\hline$\gamma_{T}$, gamma transition & & 5.246 & 5.246 & 5.246 \\
\hline$\eta$, phase slip factor & & -0.198 & -0.217 & -0.236 \\
\hline$\nu_{x}, \nu_{y}$ tunes & & $6.23,6.20$ & $6.23,6.20$ & $6.23,6.20$ \\
\hline Revolution frequency & $\mathrm{MHz}$ & 1.058 & 1.044 & 1.031 \\
\hline Ring rf harmonics & & 1,2 & 1,2 & Off \\
\hline Ring rf voltages & $\mathrm{keV}$ & 40,20 & 15,9 & Off \\
\hline Ring bunch length & meters & 165 & 174 & 248 (coast) \\
\hline rms energy spread & $\mathrm{MeV}$ & 3.5 & 2.5 & 0.5 \\
\hline$\xi_{x}, \xi_{y}$, chromaticity & & $-9.40,-7.32$ & $-9.40,-7.32$ & $\sim 0.0, \sim 0.0$ \\
\hline$\varepsilon_{x}, \varepsilon_{y}$ rms emittances & mm-mradian & 30,30 & $\sim 30, \sim 30$ & $\sim 30, \sim 30$ \\
\hline$\left\langle\beta_{y}\right\rangle$ Average beta- $y$ ring, kickers & meters & $6.36,9.30$ & $6.36,9.30$ & $6.36,9.30$ \\
\hline
\end{tabular}

their measured impedance. In Sec. III we describe the analytic and computational models employed in our analysis. Section IV describes studies performed during the SNS design in which ORBIT was successfully benchmarked with an analytic coasting beam model, and then applied to bunched-beam calculations. Section V describes the experimentally observed extraction kicker instability. Section VI presents the ORBIT simulation of the observed instability. In Sec. VII we present our conclusions. We conclude this introductory section with Table I, which compiles the important parameters of the SNS ring.

\section{SNS RING EXTRACTION KICKERS}

The accumulated beam is extracted from the SNS ring in a single turn through a Lambertson septum and then transported to the target. To perform the extraction cleanly, it is necessary to operate the ring with bunched beams. Bunched beams are created by chopping at low energy in the linac, and are maintained by three first harmonic and one second harmonic rf cavities in the ring. The ring $\mathrm{rf}$ system is described in Ref. [11]. Two kicker assemblies, each consisting of seven pulsed magnet modules, carry out the extraction by kicking the beam vertically downward. The assemblies are housed in the ring straight section opposite the injection chicane on either side of the section's upstream quadrupole doublet. The Lambertson extraction septum is located downstream of the kicker assemblies. The kickers are operated at a frequency of $60 \mathrm{~Hz}$ with a rise time of $200 \mathrm{~ns}$ and a flattop of $750 \mathrm{~ns}$. They are designed as ferrite core rectangular window frame magnets, each powered individually by its own pulse forming network (PFN) located remotely in the modulator building. In order to optimize the beam acceptance and coupling impedance, the 14 kicker magnets have various apertures, heights, and lengths. In addition, a coaxial cage was added in the extraction kicker power supply to reduce inductance in terminating resistor circuit and improve the coupling impedance, and the kicker ferrite was coated with TiN stripes to minimize eddy currents and heating. Detailed descriptions of the mechanical design of the kickers and of their pulsed power systems are given in Refs. [12,13], respectively. The structure of an extraction kicker magnet is shown in Fig. 1, which was taken from Ref. [12].

The measurements of the SNS extraction kicker impedances are thoroughly described in Ref. [5]. The horizontal and vertical impedances are different. The horizontal impedance is primarily reactive and has only a small resistive component due to the ferrite. The vertical component depends additionally on the kicker magnet termination, and it is this component that is important from the standpoint of stability. In order to address both the contributions of eddy currents in the magnet and the PFN, the impedance measurements were performed by three different methods: twin-wire measurements with external reference, twinwire measurements with shorted busbar as reference, and direct measurements at the busbar. Although measurements were carried out only for a prototype and for the
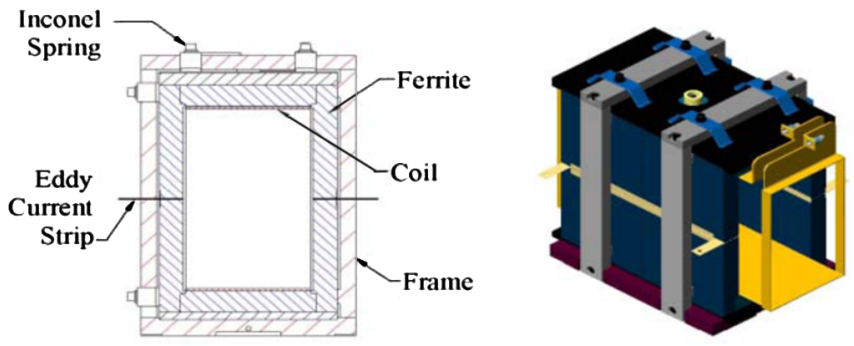

FIG. 1. Extraction kicker magnet cross section and view. 

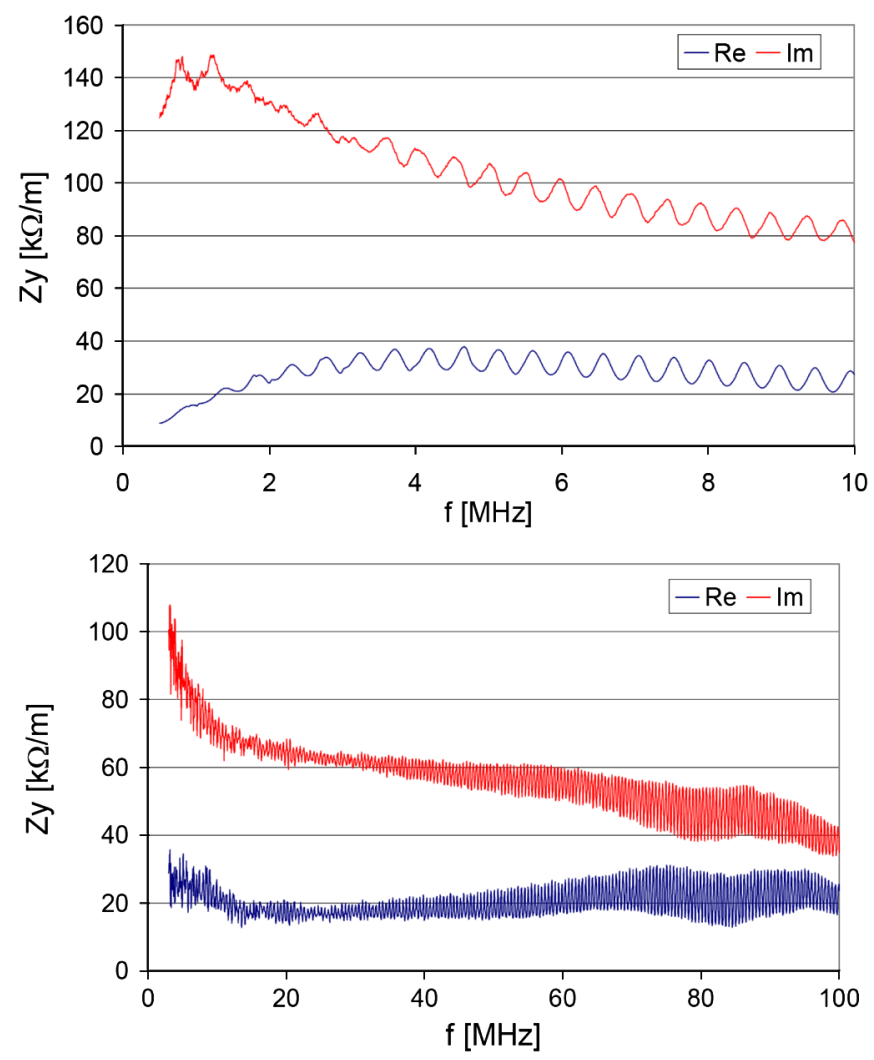

FIG. 2. Estimated coupling impedance of the SNS extraction kicker system, from Ref. [5].

smallest extraction kicker, an analytic model that incorporates the magnet geometry [14,15] was used to scale the combined measurements to estimate the overall impedance of the extraction kicker system. The result is shown in Fig. 2, which was taken from Ref. [5].

The values of the impedances used in the simulation presented in Sec. VI of this paper were based on the models presented in Refs. [14,15]. We present them here for reference in Fig. 3, which shows good agreement with the experimental estimates plotted in Fig. 2.

\section{ANALYTIC AND COMPUTATIONAL MODELS FOR TRANSVERSE STABILITY STUDIES}

In this section we present the analytic and computational models employed in these studies. Theoretical work on transverse dipole instabilities for coasting beams is a robust field. Initial models $[16,17]$ assumed linear space charge, which can be described using an impedance formulation, to develop dispersion relations that were used to determine regions of stability. A number of these models can now be found in textbooks $[1,18,19]$. Early attempts to include the space-charge nonlinearity involved the modification of these dispersion relations [20-22]. During the past decade there have been advances in understanding the effect of space-charge nonlinearity on transverse dipole instability [23-27]. In Ref. [23], Blaskiewicz applied first order
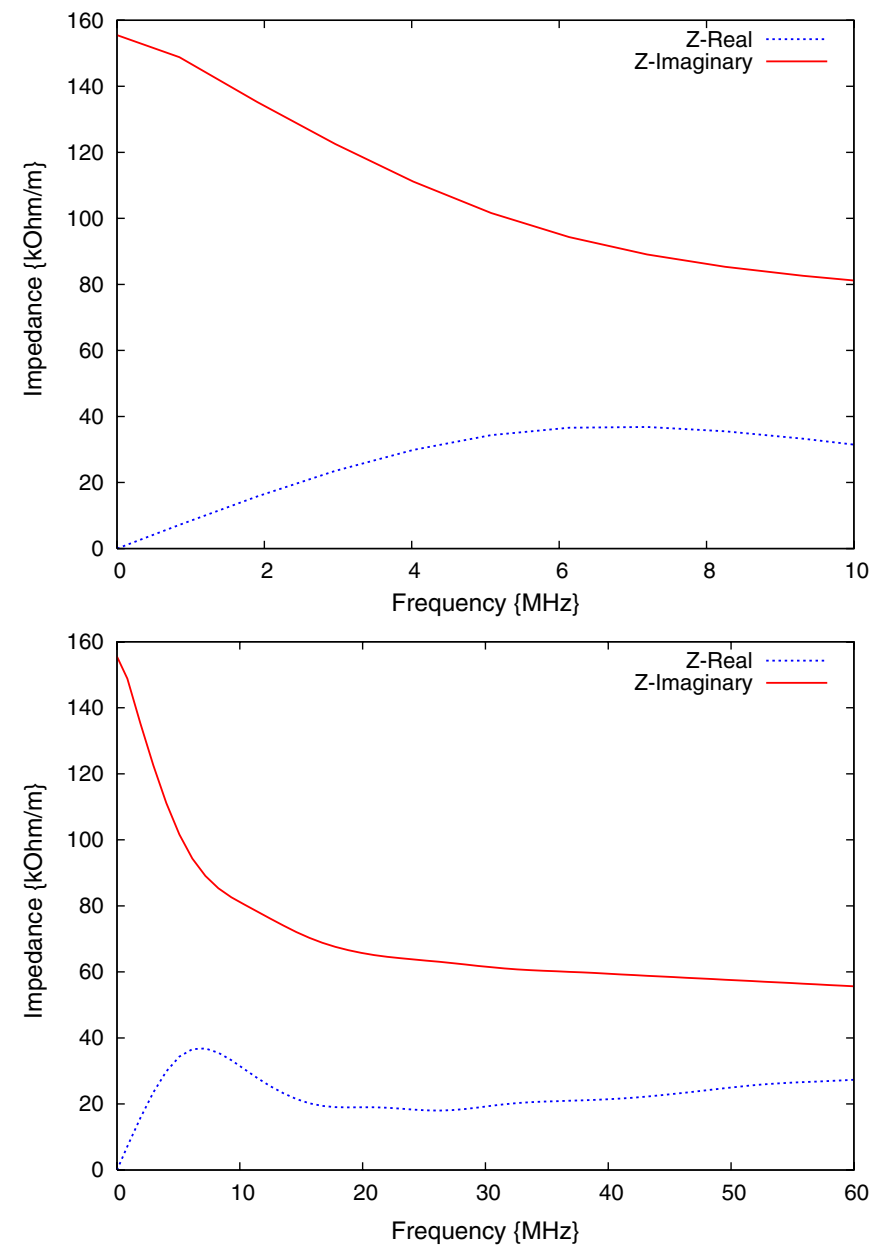

FIG. 3. Impedance of the SNS extraction kicker system used in simulations, based on Refs. [14,15].

perturbation theory to a one-dimensional Vlasov model to obtain a dispersion relation incorporating nonlinear space charge. Application of this equation to a number of model transverse beam distributions found that linear space-charge models are adequate when Landau damping arises from chromatic effects or phase slip, but that nonlinear space charge modifies stability boundaries in the presence of cubic lattice nonlinearities from octupoles or fringe fields. Starting with a two-dimensional model, Pestrikov obtained a complicated integral equation in Ref. [24]. However, in order to make further progress he dropped back to a one-dimensional approach in Ref. [25] and essentially reproduced the results of Blaskiewicz. In Ref. [26], Kornilov et al. solved Mohl's dispersion relation from Ref. [22] and compared the results with particle-incell (PIC) calculations using the code PATRIC [28]. They focused on the case of strong space-charge and octupolar lattice nonlinearities and found that nonlinearity of the space-charge force can significantly affect stability boundaries. It should be noted that their numerical calculations used a 2D space-charge solver with no longitudinal slicing. Other authors (e.g., [29]) have argued for the linear 
treatment of space charge in collective stability analysis because, unlike Landau damping from chromaticity or lattice nonlinearities, the space-charge nonlinearity moves with the beam. In Ref. [27], Burov and Lebedev delineate the domain of validity of the rigid beam instability treatment for the case where the spread of lattice frequencies is small compared to the separation between coherent and average incoherent frequencies and where the instability growth rate is slow. Using a rigid beam model applied to Gaussian beams and to chromatic and octupolar frequency spreads, they calculate Landau damping and threshold space-charge tune shifts versus coherent growth times. They find that, although rigid beam stability diagrams are not valid over most of parameter space, they are valid for most cases of practical interest.

As with coasting beams, the theoretical analysis of bunched-beam stability including synchrotron motion was first conducted a long time ago [30-32] and the resulting models have since been included in textbooks [1]. These early studies did not incorporate space charge. Balbekov [33] was the first to consider the effects of space charge in bunched beams, working in the limit that the space-charge tune shift is small compared to the synchrotron frequency. Later, Blaskiewicz [34,35] developed an analytic description of coherent modes without restrictions on the relative values of the space-charge tune shift, the synchrotron tune, and the coherent tune shift. However, this work was restricted to short-range wake forces, a square well model, and an air-bag distribution. In recent papers, Burov [36] treated general distributions and wake functions in the limit that the space-charge tune shift is much greater than the synchrotron tune and the wakedriven coherent tune shift, and Balbekov [37] considered the general problem in the limit that the instability growth rate is small compared to the synchrotron and space-charge frequency shifts. Balbekov's main conclusion is that the space-charge tune spread causes significant Landau damping which suppresses most transverse modes.

In the present work, we employ analytic calculations in two ways: first in order to benchmark the transverse impedance model in the ORBIT code; and second to estimate the magnitude of the impedance from the growth rate of the experimentally observed instability. We use a simple model for coasting beam stability in which space charge is treated through an impedance formulation. We restricted benchmarks of the model to coasting beams with transverse Kapchinskij-Vladimirskij (KV) distributions $[1,38]$, which are characterized by linear space charge forces, thus avoiding the complications of nonlinear space charge and bunched beams. Because the observed instability involves a coasting beam with corrected ( $\sim$ zero) chromaticity and significant space-charge tune shift, this simple analytic model should again be reasonably applicable. Our specific analytic formulation was discussed in detail in Ref. [2], and we briefly repeat it here, adding the effect of chromaticity.
Consider a beam with dipole moment $D(z, \delta, \tau)$ in which $z, \delta$, and $\tau$, are, respectively, the longitudinal coordinate in the bunch, the energy deviation $\delta=\frac{\delta E}{E_{\mathrm{Tot}}}=\beta^{2} \frac{\delta p}{p_{0}}$, and the commoving position coordinate $\tau=\frac{s}{\Pi}$, where $\Pi$ is the periodic length. Assume that the beam receives an impedance kick once each lattice period and undergoes betatron oscillations between kicks. The equation for $D(z, \delta, \tau)$ is $\frac{d^{2} D}{d \tau^{2}}+K(s) \Pi^{2} D=\frac{F}{\gamma m}\left(\frac{\Pi}{\beta c}\right)^{2}$, where $K(s)$ is the transverse focusing function, $F$ is the force of the impedance kick, $m$ is the particle mass, $c$ is the velocity of light, and $\beta$ and $\gamma$ are the usual relativistic factors. We define a normalized dipole moment $D_{N}=\frac{D}{\sqrt{\beta_{s} \varepsilon}}$ and a phase coordinate $\phi=\frac{1}{2 \pi \nu_{b}} \int_{s_{0}}^{s} \frac{d s}{\beta_{s}}$, where $\nu_{b}$ is the betatron tune, $\beta_{s}$ is the Courant-Snyder beta function, $\varepsilon$ is the emittance of the beam, and $s_{0}$ is some chosen reference position in the lattice. The equation for $D_{N}$ for a general periodic lattice can be written $\left(d^{2} D_{N}\right) /\left(d \phi^{2}\right)+\left[2 \pi\left(\nu_{b}+\Delta \nu_{b}\right)\right]^{2} D_{N}=$ $\frac{F}{\gamma m}\left(\frac{2 \pi \nu_{b}}{\beta c}\right)^{2} \sqrt{\beta_{s}^{3} / \varepsilon}$, where $\Delta \nu_{b}$ is the energy-dependent tune shift. We assume $\Delta \nu_{b}=\xi \frac{\delta p}{p_{0}}=\xi \frac{\delta}{\beta^{2}}$, namely, that the tune shift is due to the chromaticity, $\xi$.

To analyze this equation, some further assumptions are made. For the localized impedance, we assume a single harmonic, so that $Z_{\perp}=Z_{\perp}\left(n \omega_{0}+\omega_{b}\right)$ for lattice frequency $\omega_{0}$, betatron frequency $\omega_{b}$, and integer mode number $n$. The force with this single harmonic is then given by the equation $F=-\operatorname{Re}\left\{\operatorname{iqID}(z, \delta, \phi) Z_{\perp}\left(n \omega_{0}+\right.\right.$ $\left.\left.\omega_{b}\right) \delta_{\Pi}\left(s-s_{0}\right)\right\}$, where $q$ is the charge, $I$ is the current, $\delta_{\Pi}\left(s-s_{0}\right)$ is the periodic delta function with period $\Pi$, and $s_{0}$ is now the location of the impedance. The normalized dipole moment can then be factored into rapidly and slowly varying parts:

$$
D_{N}(z, \delta, \phi)=d_{s}(\delta, \phi) \exp \left\{i\left(2 \pi n \frac{z}{\Pi}+\int \frac{d z}{\beta_{s}}+2 \pi \nu_{b} \phi\right)\right\},
$$

where $d_{s}(\delta, \phi)$ varies slowly and is independent of $z$. We also note that the total derivative is $\frac{d}{d \phi}=\frac{\partial}{\partial \phi}+$ $\frac{d z}{d \phi} \frac{\partial}{\partial z}+\frac{d \delta}{d \phi} \frac{\partial}{\partial \delta}$, where $\frac{d z}{d \phi}=\frac{2 \pi \nu_{b} \eta \delta}{\beta^{2}} \beta_{s}, \frac{d \delta}{d \phi}=0$, and $\eta=$ $\frac{1}{\gamma^{2}}-\frac{1}{\gamma_{T}^{2}}$ is the phase slip factor. Assuming that $\delta$ and $\frac{d d_{s}}{d \phi}$ are small quantities and that $\frac{d^{2} d_{s}}{d \phi^{2}}$ is negligible, substitution of the expression for $D_{N}(z, \delta, \phi)$ into its dynamic equation yields a first order equation for $d_{s}(\delta, \phi)$.

We then make use of the fact that the collective force $F$ is small and apply the method of averaging to take the average of the force term over $K$ turns, where $K \gg 1$. We include the effect of the energy distribution in the force term by replacing the dipole moment by its integral over the unit-normalized energy distribution $g(\delta)$. The resulting equation is

$$
\frac{\partial d_{s}(\delta, \phi)}{\partial \phi}+i \Delta(\delta) d_{s}(\delta, \phi)=\chi \int_{-\infty}^{\infty} g(\delta) d_{s}(\delta, \phi) d \delta,
$$


where $\Delta(\delta)=\frac{2 \pi \delta}{\beta^{2}}\left[\left(n+\nu_{b}\right) \eta+\xi\right]$ and $\chi=\frac{-N r_{p} Z_{\perp}\left(n \omega_{0}+\omega_{b}\right)}{\gamma \beta Z_{0}} \times$ $\frac{2 \pi \beta_{s_{0}}}{\Pi}$. The remaining quantities in these equations are the number of protons $N$, the classical proton radius $r_{p}$, and the impedance $Z_{0}=377 \Omega$. Following the analysis of Ref. [1], we assume the dependence $d_{s} \propto e^{-i 2 \pi \Omega \phi}$, where $\Omega$ is complex and is expressed in units of the fundamental ring frequency. Instability occurs when the imaginary part of $\Omega$ is positive, and the stability boundary lies on the real axis in the $\Omega$ plane. Then, manipulating Eq. (1), we obtain the dispersion relation

$$
\begin{aligned}
h(n) & \equiv-\frac{N r_{p} Z_{\perp}\left(n+\nu_{b}\right) \beta^{2} E_{\mathrm{Tot}}}{2 \pi i \gamma \beta Z_{0}\left|\left(n+\nu_{b}\right) \eta+\xi\right|} \frac{2 \pi \beta_{s_{0}}}{\Pi} \\
& =\frac{1}{\int_{-\infty}^{\infty} \frac{g(\Delta E) d(\Delta E)}{\Delta E-\frac{\beta^{2} E_{\mathrm{Tot}}}{\left|\left(n+\nu_{b}\right) \eta+\xi\right|} \Omega}} \equiv \frac{1}{a(\Omega)+i b(\Omega)} .
\end{aligned}
$$

By plotting $\frac{1}{a(\Omega)+i b(\Omega)}$ as evaluated from the integral in Eq. (2) in the complex plane for real values of $\Omega$, we determine a stability diagram. By comparing this with $h(n)$ as evaluated from the first line in Eq. (2), we can determine the stability as a function of the parameters in the equation. In particular, we concern ourselves with the beam energy distribution $g(\Delta E)$, the mode number $n$, the value of the impedance, and the chromaticity $\xi$. We used the approach of Eqs. (1) and (2) to benchmark ORBIT's impedance model.

If an observed instability is well above threshold (which is our case), then the growth rate should not be overly sensitive to the beam distribution. Then, treating space charge linearly and ignoring Landau damping by assuming a delta function energy distribution, Eq. (1) can be solved to relate the impedance to the growth rate as

$$
\operatorname{Re}(Z)=\frac{2 \gamma \beta^{2} E_{0}}{\tau \beta_{s_{0}} I}
$$

where $Z$ is the impedance in $\Omega / \mathrm{m}, E_{0}$ is the proton mass in $\mathrm{eV}, \tau$ is the growth time in turns, $\beta_{s_{0}}$ is given in meters, and $I$ is the average beam current in amperes. The analytic part of our strategy in analyzing the observed instability will be to use the measured growth time in Eq. (3) to estimate the driving impedance, which can then be compared to the measured value. The assumptions used to derive Eq. (3) lead to the overestimation of the growth of a real beam with energy spread. Therefore, the experimental growth time should be longer than that from Eq. (3), and its use in Eq. (3) should result in an impedance prediction that is somewhat lower than the actual value.

The computational model used in this work is contained in the ORBIT code [4]. ORBIT is an open source particle tracking code with a wide variety of models that can be applied to many accelerator problems. Several different models are used in the benchmarks and design study results presented in Sec. IV. We use the most realistic choices to carry out the simulation of the experimentally observed instability presented in Sec. VI. For tracking purposes, one can specify any initial particle distribution. Alternatively, ORBIT has a stripper-foil model through which a statistically generated distribution can be injected turn by turn. The foil model allows for chopping (bunched beams) and also includes a Monte Carlo treatment of Coulomb, Rutherford, and nuclear elastic and inelastic scattering, both for injected and circulating beams. For injection in SNS simulations, transverse painting is carried out using time-dependent kicker elements with their actual waveforms, lengths, and locations in the lattice to obtain realistic beam distributions. At its simplest, single particle tracking can be done using the first or second order transport matrices from the MAD code (version 8) [39]. For more realistic studies, ORBIT has symplectic models for all magnetic lattice elements and drifts. Hard-edge fringe fields can be included symplectically using a formulation given by Forest [40]. ORBIT contains rf cavity models for acceleration and bunching. Collective forces are treated using particle-in-cell (PIC) methods. Longitudinal and transverse (dipole) impedances are implemented as localized elements using fast Fourier transforms (FFTs), and the values of the impedances at the desired frequencies must be provided. The longitudinal impedance model also includes the option of treating longitudinal space-charge forces as impedances using the formulation in Ref. [1]. This option is not used when the 3D space-charge model is invoked. Space charge is formulated using FFTs and methods described in Ref. [41]. There are two space-charge models. The simplest is a $2 \mathrm{D}$ model in which bunch factor effects are taken into account by weighting the applied force by the local current density. There are two variants of the 2D model: a direct force solver and a potential solver with conducting wall boundary conditions. We refer to the other space-charge model in ORBIT as the 3D model. The $3 \mathrm{D}$ model is actually a succession of $2 \mathrm{D}$ slices with a $2 \mathrm{D}$ potential solver on each slice. Again, conducting wall boundary conditions are used. Transverse and longitudinal forces are calculated by numerical differentiation of the potential. The 3D model is accurate when the longitudinal bunch scale length is much greater than the beam pipe radius, which is the case in most synchrotrons and certainly in SNS. The 3D solver is used whenever transverse impedances are considered, because the slicing incorporates the longitudinal variation of the transverse dipole moments, which is essential to the physics. The other ORBIT models employed in these calculations are a complete set of apertures, which are treated as completely absorbing, to account for beam losses during the simulation.

\section{TRANSVERSE STABILITY SNS BENCHMARK AND DESIGN STUDIES}

In this section we present results of transverse stability studies performed during the SNS design. Model 
benchmarks using the SNS lattice were carried out for coasting beams with linear KV space-charge distributions. Analytic estimates were made for stability boundaries and then compared with numerical calculations. The purpose of the benchmark calculations was actually twofold: in addition to validating ORBIT's impedance model, the benchmark calculations provided the first steps in an increasingly realistic simulation of SNS beam stability. Accordingly, the benchmark studies were extended to bunched beams, for which all calculations were numerical.

The benchmark comparisons between ORBIT and the analytic model were carried out using the SNS ring lattice for a number of scenarios. In all, we used the design tunes of $\nu_{x}=6.23$ and $\nu_{y}=6.20$. Tracking was done using, alternatively, linear MAD matrices or a fully symplectic routine. Tracking with MAD matrices incorporates Landau damping only through the phase slip factor which, for SNS (with $\gamma_{T}=5.246$ and $\gamma=2.066$ at $1.0 \mathrm{GeV}$ ) is $\eta=\frac{1}{\gamma_{T}^{2}}-\frac{1}{\gamma^{2}}=-0.198$. In addition to the phase slip factor, symplectic tracking incorporates chromatic and fringe field effects. Symplectic tracking was carried out both for natural chromaticity, which takes the value $\xi=-7.320$ in SNS, and for zero chromaticity, obtained by adjusting the sextupole fields. For the "slow mode" and natural chromaticity, the phase slip and chromatic terms in Eqs. (1) and (2) are of comparable size when $n=42$, with chromatic effects dominant at smaller values of $n$. We also did the calculations both with and without space charge, which we treated analytically as an imaginary contribution to the transverse impedance. We examined the modes $n=10$, which is in the dominant peak of the bunched-beam spectrum, and $n=25$, to benchmark ORBIT at shorter wavelength. Initially we studied a coasting KV beam with peak emittance $\varepsilon_{x}=\varepsilon_{y}=140 \mathrm{~mm} \mathrm{mrad}$, zero energy spread (a delta function distribution) and $N=3 \times 10^{14}$ protons, which is comparable to the peak density in SNS with longitudinal bunching included. These parameters give a space-charge tune shift $\Delta \nu=-0.078$. For natural chromaticity, an energy spread of $16 \mathrm{MeV}$ would be required for the chromatic tune shift to overlap the space-charge tune shift. For the delta function distribution, the analytic stability diagram predicts that all positive real impedances lead to instability, regardless of imaginary impedance values (space charge), and that negative real impedances result in stability. The ORBIT calculations, performed for $n=10$ under the variety of assumptions discussed above, were completely consistent with the analytic results. The delta function distribution can be considered a limiting case of the Lorentz distribution at zero energy spread. As a second case, we considered the Lorentz distribution, for which the dispersion relation predicts a straight-line stability diagram with the stability boundary at some positive real impedance, again independent of the imaginary value. ORBIT calculations for the Lorentz distribution again agreed with the analytic stability results. For several cases in which space charge was ignored, the agreement was precise for both $n=10$ and for $n=25$, regardless of the selection of particle transport model or the selection of zero or natural chromaticity. All cases with the impedance set above threshold, even by only a few percent, proved to be unstable while all cases with the impedance set below threshold were stable. Because of Landau damping, increasing the energy spread or the chromaticity stabilized the beams by increasing the threshold. For cases with space charge included and $n=10$, ORBIT again obtained precise agreement with the analytic model.

As a next step toward a realistic extension of the coasting beam calculations, we constructed an "SNS coasting beam" as follows: Using ORBIT, we injected a full intensity bunched beam of $1.5 \times 10^{14}$ protons over 1060 turns into the SNS ring. The dynamics included transverse painting, symplectic tracking, space charge, the ring rf focusing, and the longitudinal and transverse impedances from the extraction kickers, which dominate the ring. The beam distribution at the longitudinal center (peak) of the bunch was then used to generate a coasting beam of the same shape and intensity. The resulting transverse horizontal and vertical beam profiles are shown in Fig. 4. Although the transverse distribution is not uniform and results in nonlinear space-charge forces, the maximum incoherent space-charge tune shift is approximately $\Delta \nu \approx-0.2$, which is significantly larger than the coherent tune shift, and the linear analytic space-charge formulation should be applicable. The resulting energy distribution, shown in Fig. 5, was fit by simple functions that could be used in Eq. (2) to provide stability diagrams. The distribution was well represented by the sum of rectangular and Gaussian contributions, as shown in the figure. The bunch factor for this case in the ORBIT injection simulation was 0.4 , so we used $N=3.75 \times 10^{14}$ protons in the calculations and coasting beam simulations here.

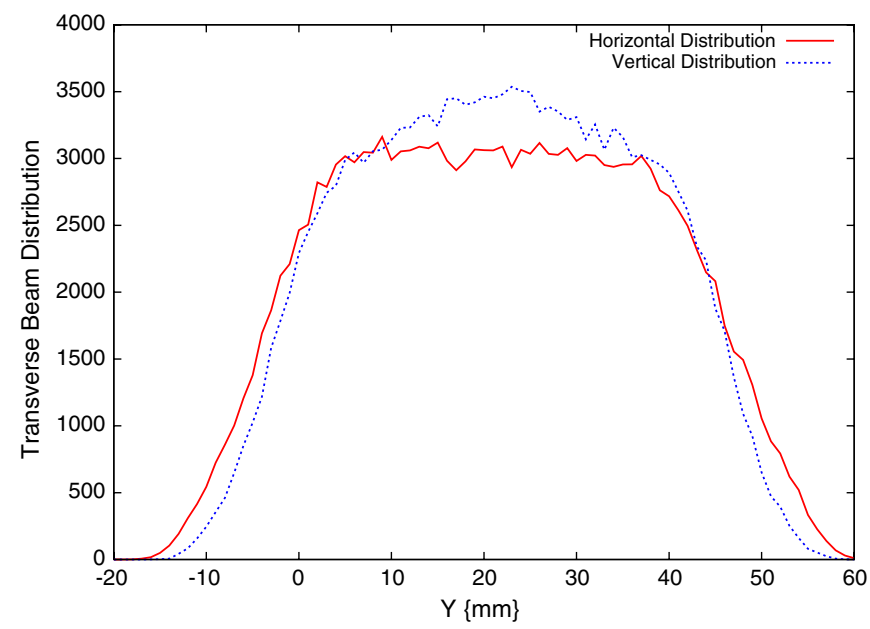

FIG. 4. Transverse profiles at beam center for the SNS coasting beam obtained from the ORBIT injection simulation. 


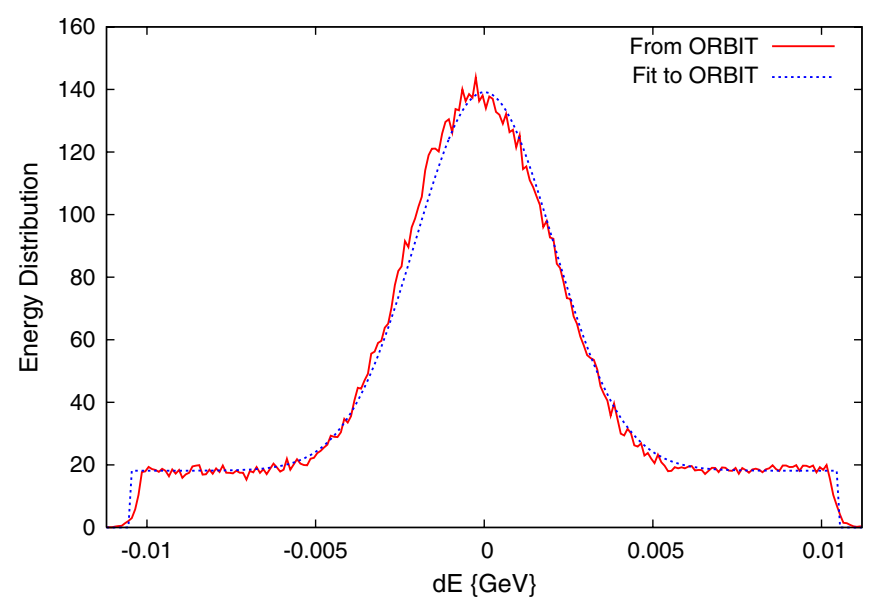

FIG. 5. The red curve shows the energy distribution of the SNS coasting beam obtained from the ORBIT injection simulation. The blue points show the fit of the analytic functions (rectangle plus Gaussian) to the simulation.

The stability diagram for the SNS coasting beam is shown in Fig. 6, which plots $\frac{1}{a(\Omega)+i b(\Omega)}$ for real values of $\Omega$. By comparing $h(n)$ in Eq. (2) to the plot, the axes can be seen to correspond to imaginary (horizontal) and to real (vertical) impedances, respectively. The region above the curve is unstable and the region below is stable. The stability diagram is valid for different values of phase slip factor, chromaticity, intensity, and mode number, but the scales depend on all these factors through Eq. (2). For $n=10$ and the SNS case considered here, at zero chromaticity a change of 0.001 on either axis in Fig. 3 corresponds to $11.2 \mathrm{k} \Omega / \mathrm{m}$, and for natural chromaticity a change of 0.001 represents $105.5 \mathrm{k} \Omega / \mathrm{m}$. In comparison, the extraction kicker impedance is $Z_{R} \sim 30 \mathrm{k} \Omega / \mathrm{m}, Z_{I} \sim$ $110 \mathrm{k} \Omega / \mathrm{m}$ at $n=10$ (about $4 \mathrm{MHz}$ for the slow mode) and the space-charge impedance is $Z_{I} \sim 3.5 \mathrm{M} \Omega / \mathrm{m}$.

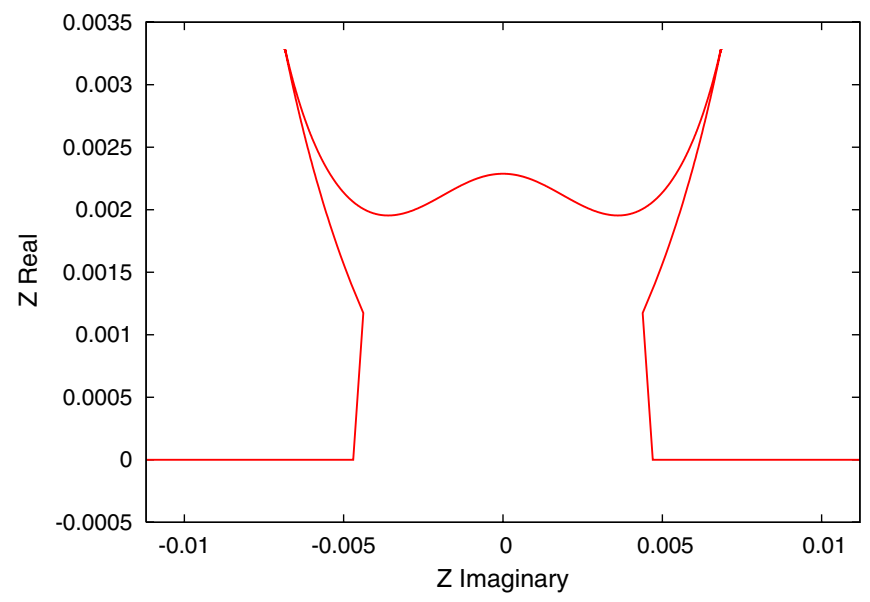

FIG. 6. The curve shows the marginal stability boundary for the energy distribution of Fig. 5 and was generated using Eq. (2). The scales depend on the detailed parameters and are described in the text.
TABLE II. SNS coasting beam stability results: $N=3.75 \times$ $10^{14}$ protons, $n=10$.

\begin{tabular}{lccc}
\hline \hline Case & $\begin{array}{c}\text { Analytic threshold } \\
(\mathrm{k} \Omega / \mathrm{m})\end{array}$ & $\begin{array}{c}\text { ORBIT stable } \\
(\mathrm{k} \Omega / \mathrm{m})\end{array}$ & $\begin{array}{c}\text { ORBIT unstable } \\
(\mathrm{k} \Omega / \mathrm{m})\end{array}$ \\
\hline 1 & 25.6 & 25 & 30 \\
2 & 25.6 & 30 & 40 \\
3 & 242 & 200 & 300 \\
4 & 0 & 0 & 10 \\
5 & & 800 & 1000 \\
\hline \hline
\end{tabular}

The analytic instability thresholds from the stability diagram in Fig. 6 have been compared with computational ORBIT results for a real impedance of $n=10$ and several cases. The results show that ORBIT is in good agreement with the analytic predictions, as shown in Table II. The cases are as follows: (1) linear MAD tracking, no space charge; (2) symplectic tracking, corrected chromaticity, no space charge; (3) symplectic tracking, natural chromaticity, no space charge; (4) linear MAD tracking, with space charge; and (5) bunched beam, natural chromaticity, no space charge. Thus, in case 1 the only Landau damping comes from the phase slip; in case 2 nonlinearities are added through sextupoles and the hard-edge fringe fields; case 3 adds the effect of natural chromaticity by turning off the sextupoles; case 4 considers space charge in the presence of phase slip; and case 5 treats the actual injected bunched beam. The ORBIT threshold for case 2 is slightly high compared with the analytic prediction due to the additional damping from the fringe field nonlinearities. Case 4 shows the destabilizing effect of space charge for coasting beam cases. Finally, case 5 was calculated using a bunched beam and, comparing with the otherwise equivalent case 3, we see that the bunched beam is much more stable. Thus, coasting beam studies, although providing the opportunity to benchmark against analytic calculations, are not relevant for normal SNS operation.

Because SNS operates with bunched beams, and because coasting beam predictions differ significantly from bunched-beam calculations, we also carried out several bunched-beam simulations with ORBIT. These were done for the design intensity of $1.5 \times 10^{14}$ protons, injected over 1060 turns, again using the design tunes. The calculations included transverse injection painting, the ring $\mathrm{rf}$ longitudinal focusing, the extraction kicker longitudinal

TABLE III. SNS bunched-beam stability results: $N=1.5 \times$ $10^{14}$ protons, (extraction kicker impedance) $\times Z$.

Case No space charge No space charge Space charge Space charge $\begin{array}{llll}\text { Stable } \times Z & \text { Unstable } \times Z & \text { Stable } \times Z & \text { Unstable } \times Z\end{array}$

\begin{tabular}{lllll}
\hline 1 & 0.5 & 0.6 & 1.5 & 2 \\
2 & 0.6 & 0.8 & 2 & 3 \\
3 & 5 & 7 & 3 & 4 \\
\hline \hline
\end{tabular}


impedance, the transverse impedance (we used 32 modes) as shown in Fig. 3, and variations on the single particle transport and presence of space-charge forces. In all cases, thresholds were obtained in terms of impedances by multiplying the measured extraction kicker impedance by varying coefficients. The results are shown in Table III, both without and with space charge. The cases are as follows: (1) linear MAD tracking; (2) symplectic tracking, corrected chromaticity; and (3) symplectic tracking, natural chromaticity. The results of case 3 show, as in the coasting beam calculations, that chromaticity leads to significant stabilization. We also see from cases 1 and 2 that, if space charge is neglected, SNS at zero chromaticity is predicted to be unstable at the extraction kicker impedance. The important columns are those including space charge. Unlike the coasting beam case in Table II, for which space charge is strongly destabilizing, the effect of space charge on the SNS bunched beam is stabilizing for the zero chromaticity case and somewhat destabilizing at natural chromaticity. Most important, these calculations predict that SNS will be stable with at least a factor of 2 to spare over the measured extraction kicker impedance. Although the stability of bunched beams is an area of active theoretical research $[33,34,36,37]$, we do not consider an analytic treatment here.

From these studies, we conclude that, for coasting beams, the ORBIT code benchmarks very well with analytic results of instability thresholds, including the effects of phase slip, chromaticity, and space charge. These beams are stabilized due to Landau damping by increasing the energy spread and/or the chromaticity. For coasting beams, when the linear treatment of space charge is appropriate, it tends to be destabilizing, as the imaginary space-charge impedance shifts the beam away from the stabilizing Landau-damped portion of the stability diagram. Also, coasting beam results are found to be less stable than those of bunched beams for otherwise similar cases. The coasting beam model predicts instability for SNS ring energy distributions and intensities, while realistic simulation with 3D space charge shows that bunched beams are stable, even for zero chromaticity. Unlike coasting beams, bunched beams are not significantly destabilized by space-charge effects. The greater stability of bunched beams is due to several factors, including the coupling of many modes and the spread of betatron tunes along the longitudinal coordinate due to vacuum chamber and bunch factor effects. Ultimately, real bunched-beam dispersion relations will be required to describe analytically our particular SNS Ring situation.

\section{THE OBSERVED EXTRACTION KICKER INSTABILITY}

Section IV shows that the Spallation Neutron Source accumulator ring was designed and constructed to be stable at the full intensity of $1.5 \times 10^{14}$ protons. Bunched-beam stability calculations using ORBIT with the extraction kicker impedance show longitudinal stability up to $8 \times$ $10^{14}$ protons [42], while Table III shows that transverse stability at $1.5 \times 10^{14}$ protons is predicted for up to 3 to 4 times the known impedance. However, for coasting beams in SNS, Table II shows that analytic and ORBIT calculations for mode number $n=10$ and $1.5 \times 10^{14}$ protons predict vertical instability when $\operatorname{Re}(Z)>0 \mathrm{k} \Omega / \mathrm{m}$ at zero chromaticity and when $\operatorname{Re}(Z) \geq 250 \mathrm{k} \Omega / \mathrm{m}$ at natural chromaticity. Because the measured impedance of the extraction kickers in the vicinity of $n=10$ is $\operatorname{Re}(Z) \sim 30 \mathrm{k} \Omega / \mathrm{m}$, it is appropriate to use coasting beams and corrected chromaticity to look for this instability experimentally.

The extraction kicker instability has been observed under these conditions in the course of high intensity beam studies. The scenario was the following: The ring tunes were set at $\nu_{x}=6.23$ and $\nu_{y}=6.20$. The chromaticity was corrected to zero and the rf buncher cavities were turned off. The choppers were also turned off so that a continuous coasting beam was accumulated. An $860 \mathrm{MeV}$ beam of $7.7 \times 10^{13}$ protons, more than $12 \mu \mathrm{C}$, was injected for 850 turns and subsequently stored until the beam was lost in the ring. The evolution of the beam was followed for 10000 turns. The observed instability began at about 1200 turns and saturated somewhat beyond 4000 turns. It was active in the transverse vertical direction with dominant harmonic at $6 \mathrm{MHz}$ and noticeable excitation in the $4 \rightarrow 10 \mathrm{MHz}$ range, as shown in Fig. 7 . Interpreting this to be a "slow" mode, the frequency is consistent with dominant harmonic $n=12$, and excitation in the range $10 \leq n \leq 16$. Because the SNS ring frequency is roughly $1 \mathrm{MHz}$ and the betatron tunes are slightly above six, mode numbers for the "slow mode" correspond roughly to the observed instability frequency in $\mathrm{MHz}$ plus six. The observed signal range agrees well with the

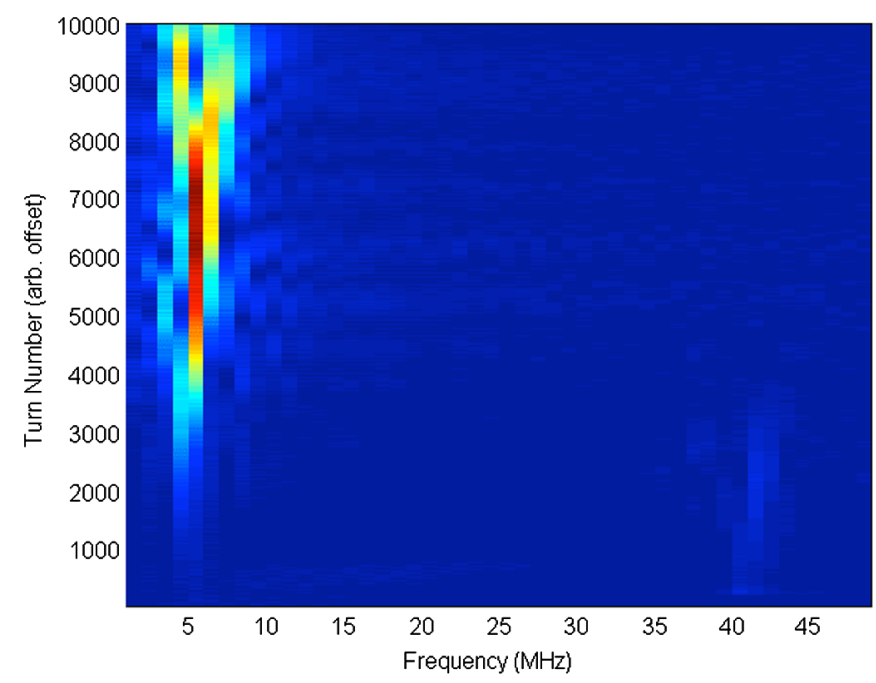

FIG. 7. Evolution of experimental turn-by-turn vertical harmonic spectrum of the extraction kicker instability. 
predicted range of dominant unstable mode numbers from the extraction kicker impedance.

The experimental results for this case have been presented in Ref. [6], and the growth rate of the $6 \mathrm{MHz}(n=12)$ harmonic was used with Eq. (3) to infer the extraction kicker impedance at that frequency. The resulting prediction of $28 \mathrm{k} \Omega / \mathrm{m}$ is in excellent agreement with the laboratorymeasured impedance of $30 \mathrm{k} \Omega / \mathrm{m}$. We have now performed a precise simulation of this case, using ORBIT and matching all known experimental details as closely as possible.

\section{SIMULATION OF OBSERVED EXTRACTION KICKER INSTABILITY}

We used the actual ring settings with $\nu_{x}=6.23$ and $\nu_{y}=6.20$ and zero chromaticity. The ring rf cavities were turned off and a continuous coasting beam of $7.7 \times 10^{13}$ protons at $860 \mathrm{MeV}$ was injected for 850 turns and stored up to 10000 turns. The injected beam rms energy spread was taken to be $0.5 \mathrm{MeV}$, consistent with observation, and the nominal SNS transverse injection painting was employed. Tracking was carried out using symplectic single particle transport, the laboratorymeasured longitudinal and transverse impedances for the extraction kickers, and the 3D space-charge model. In addition, the ORBIT foil scattering model was activated and a complete set of apertures was included to incorporate beam losses during accumulation and storage. The number of macroparticles in the simulation was $4.25 \times 10^{6}$, about double the number required for convergence of the $3 \mathrm{D}$ space-charge model.

One of the more impressive results presented in Ref. [6] was the above-mentioned agreement between the extraction kicker impedance calculated from the observed growth rate of the instability using Eq. (3) and that measured in the laboratory. There are 14 extraction kickers distributed over a length of about $10 \mathrm{~m}$ in the SNS ring. The vertical beta function at the kickers varies from a minimum of $6.4 \mathrm{~m}$ to a maximum value of $13.5 \mathrm{~m}$, with an average value of $9.3 \mathrm{~m}$. The measurement gave an experimental growth time of 1036 turns. In estimating the value of $28 \mathrm{k} \Omega / \mathrm{m}$ using Eq. (3), a value $\beta_{s_{0}}=6 \mathrm{~m}$, which is close to the average for the entire ring, was assumed. If, instead, the average value of $\beta_{s_{0}}=9.3 \mathrm{~m}$ at the extraction kickers is used, the estimated impedance is $18 \mathrm{k} \Omega / \mathrm{m}$, which is lower than the lab value of $30 \mathrm{k} \Omega / \mathrm{m}$. However, Eq. (3) was derived under the assumptions that we are far from threshold and that the energy distribution is a delta function, thus ignoring Landau damping. Both these assumptions overestimate the growth of a real beam with energy spread. Therefore, we expect the experimental growth time to be longer than that from Eq. (3), and its use in Eq. (3) should result in an impedance prediction that is somewhat lower than the actual value.

The ORBIT simulation was carried out with a single extraction kicker impedance node using the experimentally

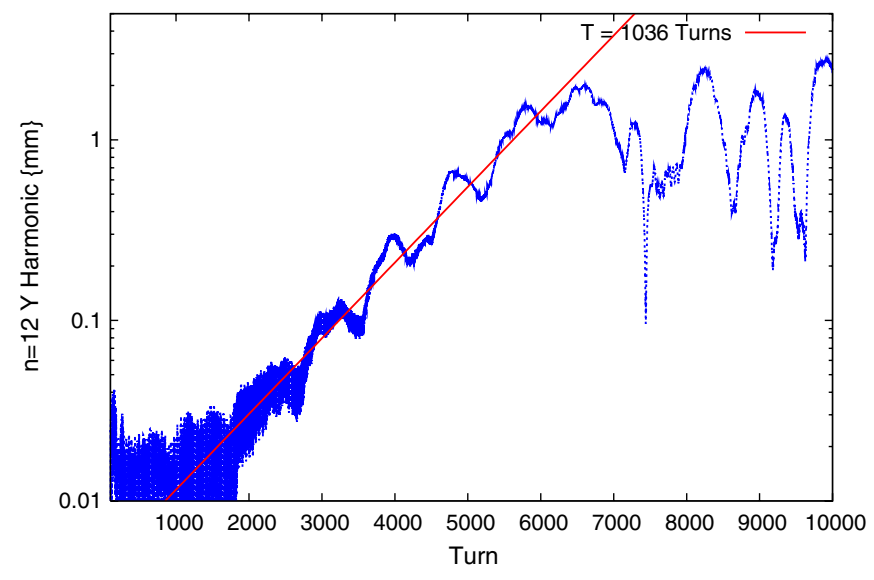

FIG. 8. Vertical $n=12$ harmonic (in blue) versus turn number in the ORBIT extraction kicker instability simulation. The red line depicts an exponential growth time of 1036 turns.

measured impedance values from Fig. 3 and placed at a position among the extraction kickers where the beta function satisfies $\beta_{s_{0}}=9.3 \mathrm{~m}$. The result, shown in Fig. 8, is an exponential growth time for the $n=12$ harmonic that is completely consistent with the measured time of 1036 turns. This impressive result is an important testimony to the necessity of getting all the details correct when performing a quantitative comparison between experiment and simulation. In reaching the result of this simulation, we made a number of false starts [8-10]. Erroneous assumptions included the use of chopped beams, the use of (too large) impedances from a previous design of the extraction kickers, and the placement of the impedance node at the geometric center of the extraction kickers rather than at a location with the average beta function. With hindsight, mistakes such as these appear to be foolish. However, in simulating a complicated particle accelerator, there are many details, each of which can affect the results. As

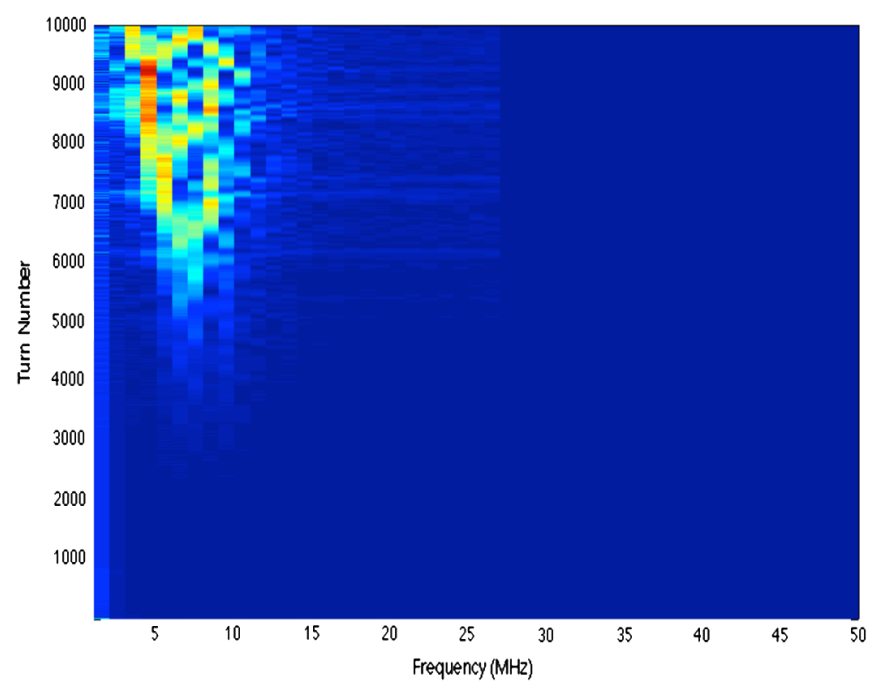

FIG. 9. Evolution of simulated turn-by-turn vertical harmonic spectrum of the extraction kicker instability. 
each of these errors was rectified, the simulation improved, until we now achieve the correct growth rate for the instability.

We have completed the calculation to 10000 turns. The evolution of the experimental and simulated turn-by-turn spectra shown in Figs. 7 and 9, respectively, display activity over a similar range of frequencies, although the simulation shows somewhat more spreading than the experiment. Also, most of the activity occurs after 5000 turns, which is after the completion of the linear growth. The experimental signals were extracted from an FFT of high bandwidth beam position monitor data. The simulated signals are harmonics of the beam centroid oscillations, measured in millimeters. Thus, although the agreement between simulation and experiment is excellent in the linear growth phase of the instability, as shown in Fig. 3, it is only qualitative during the nonlinear latter stages of the evolution. During this time other effects, such as beam loss, can complicate the comparison.

\section{CONCLUSIONS}

During one of the dedicated high intensity accelerator physics studies in SNS, the extraction kicker instability was observed experimentally and documented. Subsequently, a simple theoretical analysis using Eq. (3) was applied to the observed growth rate of the dominant $n=12$ harmonic to give an estimate of the extraction kicker impedance that was in reasonable agreement with the measured value $[5,6]$. In this paper we presented a careful simulation of the observed extraction kicker impedance instability using the ORBIT code and the detailed parameter values from the experiment. In particular, we considered a coasting beam, accumulated with the observed lattice settings, injection scenario, beam intensity, energy spread, and chromaticity. We also included space charge, foil scattering, and beam losses during injection and storage of the beam for 10000 turns. Before presenting the simulation results, we showed the excellent benchmarks of ORBIT with theory for a variety of SNS cases including the effects of space charge and Landau damping due to energy spread, phase slip, and chromaticity as well as the predictions of ORBIT for SNS stability of coasting and bunched-beam cases. In the simulation of the experiment, we found that the growth rate of the $n=12$ harmonic agrees precisely with the experimentally observed value. Comparison of the spectral evolution of the experiment and simulation out to 10000 turns shows qualitatively similar results, with harmonics in the range $n=10-16$ dominant.

[1] A. Chao, Physics of Collective Beam Instabilities in High Energy Accelerators (John Wiley and Sons, New York, 1993).
[2] V. V. Danilov and J. Holmes, in Proceedings of Halo '03, ICFA Advanced Beam Dynamics Workshop, Montauk, NY, 2003.

[3] J. A. Holmes, V. Danilov, and L. Jain, in Proceedings of the 21st Particle Accelerator Conference, Knoxville, 2005 (IEEE, Piscataway, NJ, 2005), TPAT032.

[4] J. A. Holmes, S. Cousineau, V. V. Danilov, S. Henderson, A. Shishlo, Y. Sato, W. Chou, L. Michelotti, and F. Ostiguy, in The ICFA Beam Dynamics Newsletter (2003), Vol. 30.

[5] H. Hahn, BNL/SNS Technical Note No. 135, Brookhaven National Laboratory, Upton, NY, 2004.

[6] V. Danilov, S. Cousineau, A. Aleksandrov, S. Assadi, W. Blokland, C. Deibele, S. Henderson, J. Holmes, M. Plum, and A. Shishlo, in Proceedings of the 41st ICFA Advanced Beam Dynamics Workshop on High-Intensity, HighBrightness Hadron Beams (HB2006), Tsukuba, Japan, 2006.

[7] S. Cousineau, in Proceedings of the 42nd ICFA Advanced Beam Dynamics Workshop on High-Intensity, HighBrightness Hadron Beams (HB2008), Nashville, 2008.

[8] J. A. Holmes, S. Cousineau, V. Danilov, and A. Shishlo, in Proceedings of the 11th European Particle Accelerator Conference, Genoa, 2008 (EPS-AG, Genoa, Italy, 2008).

[9] J. A. Holmes, S. Cousineau, V. Danilov, M. Plum, and A. Shishlo, in Proceedings of the 42nd ICFA Advanced Beam Dynamics Workshop on High-Intensity, High-Brightness Hadron Beams (HB2008), Nashville, 2008 (Ref. [7]).

[10] J. A. Holmes, S. Cousineau, V. Danilov, and Z. Liu, in Proceedings of the 23rd Particle Accelerator Conference, Vancouver, Canada, 2009 (IEEE, Piscataway, NJ, 2009).

[11] M. Blaskiewicz, J. M. Brennan, J. Brodowski, J. Delong, M. Meth, K. Smith, and A. Zaltsman, in Proceedings of the 19th Particle Accelerator Conference, Chicago, Illinois, 2001 (IEEE, Piscataway, NJ, 2001).

[12] C. Pai, D. Davino, H. Hahn, H. Hseuh, Y. Lee, W. Meng, J. Mi, J. Sandberg, N. Tsoupas, J. Tuozzolo, D. Warburton, and W. Zhang, in Proceedings of the 20th Particle Accelerator Conference, Portland, OR, 2003 (IEEE, New York, 2003), p. 2147.

[13] W. Zhang, J. Sandberg, H. Hahn, J. Mi, C. Pai, Y. Tan, N. Tsoupas, J. Tuozzolo, D. Warburton, and J. Wei, in Proceedings of the 9th European Particle Accelerator Conference, Lucerne, 2004 (EPS-AG, Lucerne, 2004), p. 1810.

[14] D. Davino and H. Hahn, Phys. Rev. ST Accel. Beams 6, 012001 (2003).

[15] D. Davino, J-L. Mi, and N. Tsoupas, BNL/SNS Technical Note No. 112, Brookhaven National Laboratory, Upton, NY, 2002.

[16] L. J. Laslett, V. K. Neil, and A.M. Sessler, Rev. Sci. Instrum. 36, 436 (1965).

[17] A. G. Ruggiero and V. G. Vaccaro, CERN Report No. ISRTH/68-33, 1968.

[18] A. Hofmann, CERN Accelerator School, Rhodes, Greece, 1993 (CERN 95-06 V1, p. 275).

[19] K-Y. Ng, U.S. Particle Accelerator School, SUNY Stony Brook, New York, 2000 (FERMILAB-Conf-00/142T).

[20] D. Mohl and H. Schonauer, in Proceedings of the IX International Conference on High Energy Accelerators, Stanford, 1974 (AEC, Washington, DC, 1974), p. 380. 
[21] D. Mohl, Part. Accel. 50, 177 (1995).

[22] D. Mohl, CERN Report No. PS 95-08 (DI) 1995.

[23] M. Blaskiewicz, Phys. Rev. ST Accel. Beams 4, 044202 (2001).

[24] D. V. Pestrikov, Nucl. Instrum. Methods Phys. Res., Sect. A 562, 65 (2006).

[25] D. V. Pestrikov, Nucl. Instrum. Methods Phys. Res., Sect. A 578, 65 (2007).

[26] V. Kornilov, O. Boine-Frankenheim, and I. Hofmann, Phys. Rev. ST Accel. Beams 11, 014201 (2008).

[27] A. Burov and V. Lebedev, Phys. Rev. ST Accel. Beams 12, 034201 (2009).

[28] O. Boine-Frankenheim and V. Kornilov, in Proceedings of the International Computational Accelerator Physics Conference (ICAP2006), Chamonix Mont-Blanc, 2006.

[29] V. Balbekov, Report No. FERMILAB-FN-0782-AD, 2006.

[30] C. Pellegrini, Nuovo Cimento A 64, 447 (1969).

[31] M. Sands, SLAC Reports No. TN-69-8 and No. TN-69-10, 1969.

[32] F. Sacherer, CERN Report No. CERN-SI-BR-72-5, 1972.

[33] V. Balbekov, Zh. Tekh. Fiz. 46, 1470 (1976) [Sov. Phys. Tech. Phys. 21, 837 (1976)].
[34] M. Blaskiewicz, Phys. Rev. ST Accel. Beams 1, 044201 (1998).

[35] M. Blaskiewicz, Phys. Rev. ST Accel. Beams 6, 014203 (2003).

[36] A. Burov, Phys. Rev. ST Accel. Beams 12, 044202 (2009).

[37] V. Balbekov, Phys. Rev. ST Accel. Beams 12, 124402 (2009).

[38] I. M. Kapchinskij and V. V. Vladimirskij, in Proceedings of the International Conference on High Energy Accelerators (CERN, Geneva, 1959), p. 274.

[39] H. Grote and F. C. Iselin, Report No. CERN/SL/90-13 (AP) (Rev. 5) 1996.

[40] E. Forest, Beam Dynamics, A New Attitude and Framework (Harwood Academic Publishers, Amsterdam, 1998).

[41] R. W. Hockney and J. W. Eastwood, Computer Simulation Using Particles (Institute of Physics Publishing, Philadelphia, 1999).

[42] K. Woody, J. A. Holmes, V. Danilov, and J. D. Galambos, in Proceedings of the 19th Particle Accelerator Conference, Chicago, Illinois, 2001 (Ref. [11]), p. 2057. 